\title{
A modelling formalism for distributed manufacturing simulator
}

\author{
S. Fujiil ${ }^{l)}$, T. Kaihara ${ }^{2)}$, H. Morita ${ }^{3)}$, A.Ouchi ${ }^{\text {l) }}$ \\ ${ }^{1)}$ Kobe University, Faculty of Engineering \\ Rokkodai, Nada, Kobe, 657-8501, Japan \\ Tel: +81-78-803-6239 \\ Fax: +81-78-803-6391 \\ e-mail: fujii@seg.kobe-u.ac.jp, ouchi@kaede.seg.kobe-u.ac.jp \\ ${ }^{2)}$ University of Marketing and Distribution Sciences, Faculty of \\ Information Science \\ e-mail: kaihara@umds.ac.jp \\ ${ }^{3)}$ Kobe University, Graduate School \\ E-mail:morita@seg.kobe-u.ac.jp
}

\begin{abstract}
Manufacturing system model specification varies in each simulation model, due to the lack of generalised manufacturing system modelling formalism in conventional object-oriented modelling approaches. In order to overcome those difficulties, a procedural modelling formalism of distributed manufacturing simulator is proposed. Because a standardised modelling template with IDEF 0 realises procedural modelling via a logical decomposition of the objective system, the proposed approach facilitates multi-stage manufacturing system planning.
\end{abstract}

\section{Keywords}

Manufacturing system modelling, Manufacturing simulation, Object-oriented programming, Distributed simulation, IDEF 0

\section{INTRODUCTION}

The integration of manufacturing system now in progress made it crucial to operate the system efficiently as a whole. Distributed simulation system is one of the most important concepts to support the effective operation for a large-scaled manufacturing system (Fujii, 1992, 1994, 1996). In the previous works, we have proposed i) simulation engine architecture and ii) synchronisation mechanism, in distributed environment. However, the modelling problem of each sub-model in- 
volved in distributed manufacturing simulator still exists, and flexible and extensible modelling methodology is essential to distributed manufacturing domain.

Since object-oriented paradigm is originated from simulation language, it is well known that the integration between manufacturing system modelling and object-oriented programming is effective for its modularity, its extendibility, and its reusability (Kaihara, 1993). Manufacturing simulation model, however, still depends on the model programmer even in object-oriented paradigm, due to the lack of generalised manufacturing system modelling formalism. In order to overcome those difficulties a procedural modelling formalism of distributed manufacturing simulator based on object-oriented paradigm is proposed as a basic study for automatic simulation program generator in this paper. The proposed manufacturing modelling formalism has the following three major ideas:

i) Separated modelling of material flow and information flow by IDEF 0 (Integrated CAM DEFinition 0) description

ii) Class definition extracted from IDEF 0

iii) Manufacturing system model template (object class library) with transparency

This paper investigates the effectiveness of the newly proposed ideas in semiconductor fabrication line as a case study, and indicates the advantages of the formalism in distributed simulation environment from a practical point of view.

\section{SEPARATED MODELLING OF MATERIAL FLOW AND INFORMATION FLOW}

\subsection{IDEF 0 description}

IDEF 0 is a method designed to model the decisions, actions, and activities of a system or organisation (Ishibashi, 1997). IDEF 0 was derived from a wellestablished graphical language, the Structured Analysis and Design Technique (SADT). The United States Air Force commissioned the developers of SADT to develop a function modelling method for analysing and communicating the functional perspective of a system. In IDEF 0 Activities can be described by their Inputs, Outputs, Controls, and Mechanisms. Effective IDEF 0 models help to organise the analysis and the modelling of large scale manufacturing systems.

\subsection{Constructional rules for separated modelling}

IDEF 0 originated from SADT includes hierarchical diagram description concept. Since the description of the activities of a system can be easily refined into greater and greater detail until the model is as descriptive as necessary for the decisionmaking task, IDEF 0 perfectly fits inherited framework in object-oriented programming. IDEF 0 analyses generalised manufacturing model structure, by which standard manufacturing class definition is extracted procedurally. 


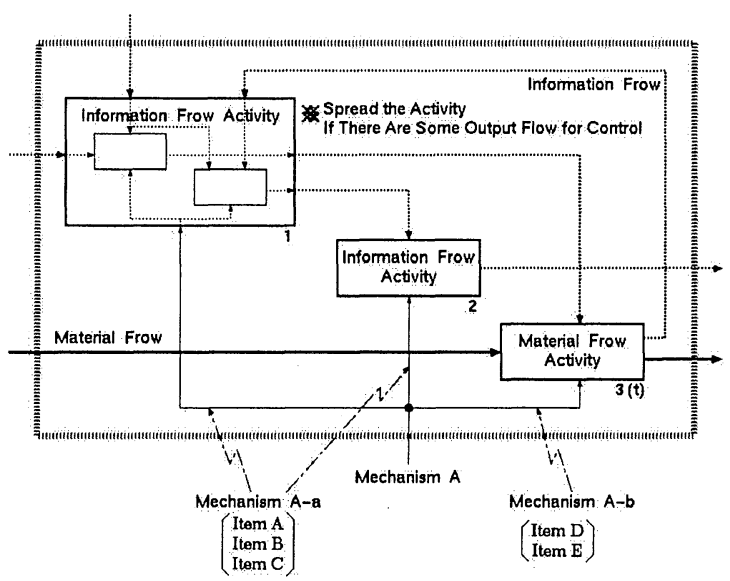

Figure 1 Separated modelling using IDEF 0

Figure 1 illustrates the proposed idea of separated modelling between material flow and information flow in IDEF 0 . Thick arrows and dotted arrows indicate material flows and information flows, respectively. There are also two types of Activities defined, material flow activity and information flow activity, in our approach. In the proposed formalism, the following constructional rules are introduced:

(1) The number of Inputs into each Activity can not be more than one. Only information flow activity is allowed to have no Input.

(2) Mechanism name must be unique between any material flow and information flow. For example, Mechanism names both on Activity 1 and on Activity 3 are defined differently in figure 1.

(3) The number of Output from an Activity into other Controls must be one at most. Any Activity that has multiple Outputs must be decomposed.

(4) Attributes required by each Mechanism must be described in brace.

(5) Activity is classified into three types with respect to data flow processing.

d: Activity with detailed data flow beyond IDEF 0 definition

t: Activity with time delay in its data processing

w: Activity with data buffer for synchronisation with other Activities

\section{CLASS DEFINITION}

\subsection{Class and method extractions from IDEF 0}

Generally each element which constructs the manufacturing system is defined as class in object-oriented manufacturing model. Because Mechanism represents the resource to execute Activity, Mechanism in IDEF 0 corresponds to Class in objectoriented programming under general manufacturing model. 
In our formalism, each class is extracted from Mechanism in IDEF 0 , and the class is named after the corresponding Mechanism. Additionally each Activity designated by the Mechanism is defined as method in the class. For example, class 'A$\mathrm{a}^{\prime}$ with 2 types of method and class 'A-b' with a method are extracted in figure 1.

\subsection{Attributes in Class}

After classes and methods are extracted, it is necessary to define attributes in each class procedurally according to object-oriented programming manner. Basically all the attributes defined in Mechanism are automatically regarded as attributes in the extracted class. Input in IDEF 0 is also defined as Attribute, in case a Control brings one Input in pair between any two activities (figure 2(a)). However, if a Control and an Input have different senders i.e. different routes (figure 2(b)), it is difficult to define attributes in a systematic manner as in figure 2(a).

Attribute definition mechanism in different senders is shown in figure 3 . The following six steps are carried out to define standard attributes.

Step 1: Output data from Method A is reserved as attributes in the class.

Step 2: Method A sends a control signal into other methods.

Step 3: Method B in class Y finally gets a control signal from other methods.

Step 4: Method B invokes pop up method in class X.

Step 5: Reserved original data is fetched from attributes.

Step 6: Pop out method hands the data to Method B.

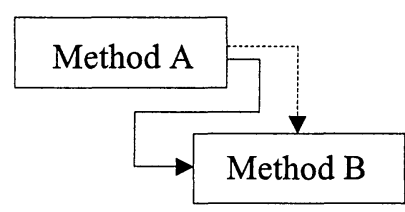

(a) Common sender

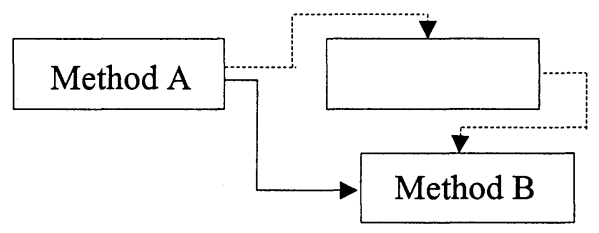

(b) Different senders

Figure 2 Example of Activity relations in IDEF 0

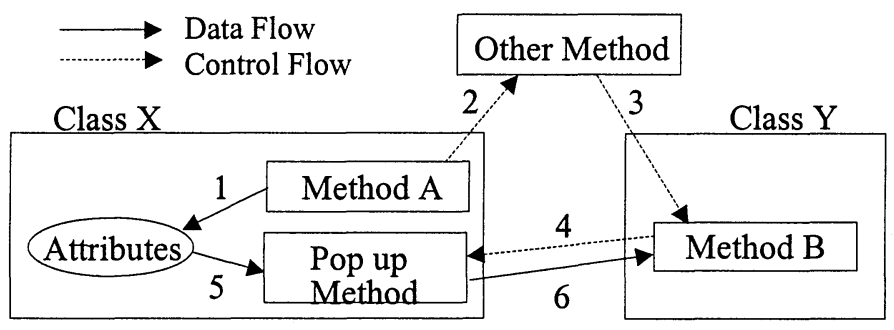

Figure 3 Attribute definition mechanism

Finally class attributes are extracted from IDEF 0 procedurally as follows:

i) all the attributes defined in Mechanism

ii) all the data defined in Input 
iii) all the attributes to transfer or reserve output data

As a result, Activity and Mechanism information in the IDEF 0 description are rationally transformed into method and class definitions in object-oriented manufacturing model respectively in the proposed formalism.

\section{MODEL TEMPLATE DEFINITION}

\subsection{Phased model}

The development of an integrated manufacturing system involves several planning phases. They are consecutive and the information required in each phase differs in its accuracy. We classify the system development into four major planning phases.

Plan Examination phase (Phase 1) deals with the top level manufacturing strategy based on marketing research. Macro production plan is estimated under rough production process flow in this phase. The top-level strategy is followed by concrete manufacturing system planning in Fundamental Design phase (Phase 2). Manufacturing system specification is authorised roughly in this phase. Detailed Design phase (Phase 3) are investigated mainly by manufacturing engineers. Detailed data in static conditions or simple control logics are examined in this phase. Once the operation starts, sophisticated dynamic operations, such as scheduling or work flow control, are essential to settle the manufacturing operation and manufacturing system model plays an important role to investigate operational logic under dynamic conditions in Operational phase (Phase 4). Model template is the object class library in which all the facilities in the manufacturing system are included and classified into tree structure according to the planning phase.

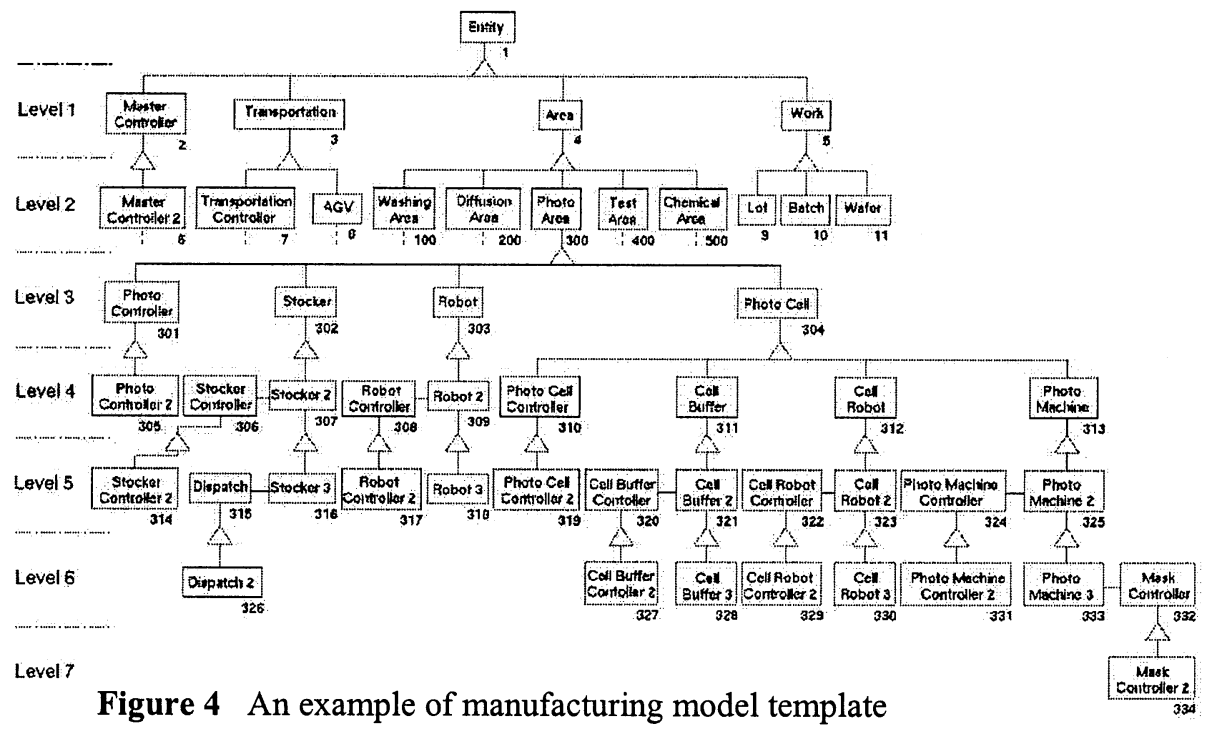

In IDEF 0 , the description of the activities of a system can be easily refined into greater and greater detail until the model is as descriptive as necessary for the 
decision-making task at hand. The hierarchical nature of IDEF 0 facilitates the ability to construct general raw manufacturing models. Beginning with the topmost activity, the detailed manufacturing model can be described via a logical decomposition. The process can be continued recursively to the desired level of detail. When an existing manufacturing system is being analysed and modelled, observed hierarchical activities can be described and transformed procedurally into manufacturing model template. As an example, a model template for semiconductor fabrication line defined by the proposed formalism is shown in figure 4.

In distributed simulator all the facility classes defined in level 1 are basically correspond to sub models. For example, Area class, transportation class and master controller class will be implemented on different CPUs in figure 4 .

Since there generally exist several sub models with various planning phases in a distributed manufacturing simulator, a mechanism, which handles those sub models transparently, is required. The class sorting mechanism with level switch facilitates transparent modelling in the distributed simulator. The mechanism maintains the data communication between different levels of classes and sorts out incoming messages into the corresponding classes.

Level switch shown in figure 5 is defined as an attribute in all the classes. Class sorting mechanism is able to realise the level of detail with the level switch.
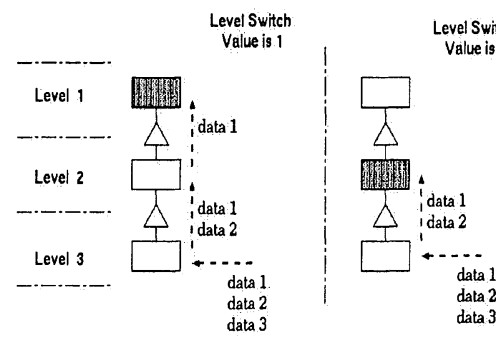

Figure 5 Level switch

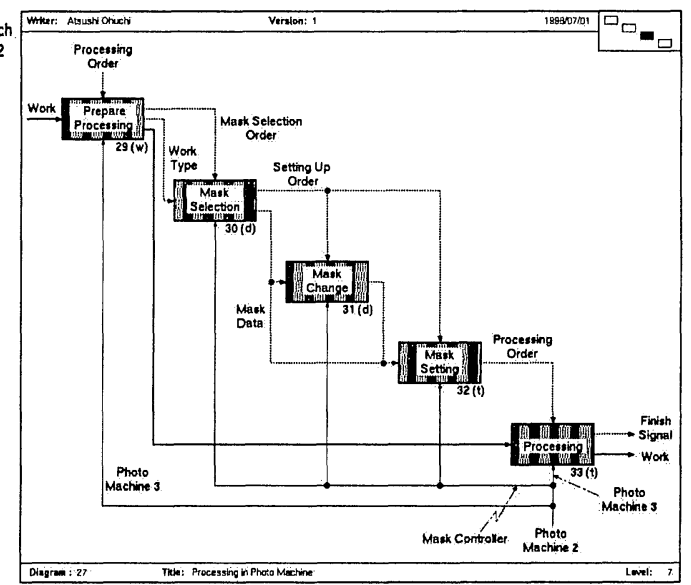

Figure 6 IDEF 0 description about photo machine

\section{A CASE STUDY}

\subsection{Semiconductor fabrication system}

The proposed formalism is applied into semiconductor fabrication modelling for a distributed virtual factory that consists of several area simulators implemented in different CPUs. Figure 6 shows IDEF 0 description about the processing in photo 
machine at phase 3 . Two types of class concerned with photo machine are extracted with five methods in this figure.

Acquired model template is already shown in figure 4. It is obvious that IDEF 0 structure was decomposed into 7th layer to describe Mask Controller 2 in this figure. Detailed control logics considering dynamic conditions, such as machine faults, are implemented into all the classes defined at the bottom in figure 4 .

\subsection{Simulation results}

Simulation results in photo process area are shown in table 1. All areas in the semiconductor fabrication model are commonly defined in phase 4 at the first row (Phase 4) in the table. At the second row (Phase $1 \& 4-1$ ), only the photo area is defined in phase 4 , while other areas are defined in phase 1. Although we can observe the difference between the simulation results due to the model variation, it has been proved that the proposed formalism can handle various models in phase transparently. In Phase $1 \& 4-2$, simulation parameters are intentionally modified to obtain the equivalent result in the number of input works as comparison. The result about queue length in photo area is obviously improved in this table.

Table 1 Simulation results in photo process area

\begin{tabular}{ccc}
\hline Modelling phase & The number of input works & Queue length \\
\hline Phase 4 (All) & 17 & 0.9411 \\
Phase $1 \& 4-1$ & 22 & 0.7272 \\
Phase $1 \& 4-2$ & 17 & 0.8750 \\
\hline
\end{tabular}

\subsection{Discussions}

In the case study the proposed formalism has been proved to establish general modelling template with IDEF 0 description. Standardised manufacturing class definition is extracted procedurally in the formalism. As a result, the structured modelling template enables phased manufacturing system modelling via a logical decomposition according to manufacturing system planning.

The class sorting mechanism with level switch realised transparent modelling and execution in the distributed simulator with a variety of models in detail. The mechanism maintains the data communication between different levels of classes and sorts out incoming messages into the appropriate classes transparently.

\section{CONCLUSIONS}

A new modelling formalism based on object-oriented paradigm has been proposed in this paper. All the classes, methods and attributes in object-oriented manufacturing model are logically extracted from IDEF 0 description, and are defined procedurally in the formalism. Since the model definition process in IDEF 
0 corresponds to manufacturing planning phases via a logical decomposition, the constructed manufacturing model template is standardised in each planning phase. General manufacturing model for a distributed simulation is attainable by the formalism. Model transparency in the manufacturing model template has been confirmed by the case study on semiconductor fabrication line. The distributed simulator, which consists of various manufacturing sub models in detail, has successfully evaluated multi-phased consecutive manufacturing plans.

As the result, our modelling formalism has proved capable of generalisation and extensibility coping with the several modelling demands. As a clear extension of the research, we currently focus on automatic simulation program generator based on the formalism.

\section{REFERENCES}

Fujii, S. (1992) Simulation in Manufacturing Systems and Its Application, Journal of The Information Processing Society of Japan, Vo.33, No.3, 240-247.

Fujii, S., et al (1994), Distributed Simulation Model for Computer Integrated Manufacturing, Proc. 1994 Winter Simulation Conference, 946-953.

Fujii, S., et al (1996) A Study on a Distributed Simulation for the Evaluation of Large Manufacturing System, Proc. S.M.Wu Symposium, Vol.II, 7-12.

Ishibashi, T. (1997) IDEF: A method for BPR, Operations Research, ORSJ, 201209.

Kaihara, T., et al (1993) Object-Oriented Flexible and Integrated Manufacturing System Modelling, Proc. of Computers in Design, Manufacturing, and Production, 312-319.

\section{BIOGRAPHY}

Susumu Fujii is a Professor of Computer and Systems Engineering at Kobe University, Kobe, Japan. He received B.E. and M.E. from Kyoto University, Japan, and Ph.D. from the University of Wisconsin, U.S.A., in 1965, 1967 and 1971 , respectively. He is a member of various academic societies such as JSPE, JSME, ORSJ, IFIP, INFORMS and others.

Toshiya Kaihara is an associate Professor at University of Marketing and Distribution Sciences. He received B.E. and M.E. from Kyoto University, Japan, and Ph.D. from Imperial College, University of London, UK, in 1983, 1985 and 1994, respectively. He is a member of ISCIE, IEEJ, IFIP, IEEE, and others.

Hiroshi Morita is an associate Professor of Graduate School of Kobe University, Kobe, Japan. He received B.E. and M.E. from Osaka University, Japan, and D.E. from Kyoto University, Japan, in 1983, 1985 and 1992, respectively. He is a member of ORSJ, JSPE, ISCIE, INFORMS and others.

Atsushi Ouchi received B.E. and M.E. from Kobe University, Japan, in 1997 and 1999, respectively and is at Sanyo Electric Co., Japan from April, 1999. 